\title{
An Intelligent Digital Learning Platform to Enhance Digital Health Literacy
}

\author{
https://doi.org/10.3991/ijet.v17i04.27907
}

\author{
Kawitsara Eumbunnapong ${ }^{1}$, Panita Wannapiroon ${ }^{2}$, Phisit Pornpongtechavanich ${ }^{3(\varpi)}$ \\ ${ }^{1}$ Faculty of Medicine Siriraj Hospital, Mahidol University, Bangkok, Thailand \\ ${ }^{2}$ King Mongkut's University of Technology North Bangkok, Bangkok, Thailand \\ ${ }^{3}$ Rajamangala University of Technology Rattanakosin, Wang Klai Kangwon Campus, \\ Prachuap Khiri Khan, Thailand \\ phisit.kha@rmutr.ac.th
}

\begin{abstract}
Nowadays spreading of COVID-19, people are more at home and less physically active in the long run, which may affect their health. Moreover, currently, there are many facilities that make human life more comfortable. Various technologies are being used to assist with daily activities. Including the perception of news has changed to the perception of news through the Internet. For this reason, this research has been working about intelligent digital learning platform to enhance digital health literacy, the research objectives are as follows. (1 synthesize documents and international research of intelligent digital learning platform to enhance digital health literacy (2design an intelligent digital learning platform to enhance digital health literacy ( 3 assess the suitability of intelligent digital learning platform to enhance digital health literacy.Will use ten experts as assessors. Those ten peoples are divided into five computer engineering technology experts and five medical technology experts who have more than five years of related experience. The research instruments were a suitability assessment form for intelligent digital learning platform to enhance digital health literacy .The results of this research found that the intelligent digital learning platform to enhance digital health literacy, was developed and it was appropriate.
\end{abstract}

Keywords - digital learning platform, intelligent technology, intelligent learning platform, digital health literacy

\section{$1 \quad$ Introduction}

Thailand's health system is facing the challenge of a rapidly aging society. The major problem for the elderly is health. Those who lack health rehabilitation are likely to suffer from chronic illnesses. Since the coronavirus (COVID-19) pandemic reached Thailand in 2020, those infected have had serious symptoms, such as fever and respiratory failure. In some cases, patients have died. The Thai government has declared a state of emergency, prohibited people from gathering in large groups and made the wearing of surgical masks outside mandatory. They have focused on working from home. This has had a huge impact on the normal lives of people and led to the advent of a new normal. This reflects the change in living behavior, work, and education. People increasingly 
rely on internet access and the use of digital technology to receive health information. While the health insurance system in Thailand is incompatible. The basic benefits are also different in detail, especially as the health personnel and medical technology are concentrated in certain areas, such as Bangkok and other central and urban areas. Moreover, for the recording of big data, some entities lack data sharing, especially between their outposts and the support agencies. This makes data redundant, increasing the risk of errors. Information tends to be inaccurate and incomplete. [1] There are also challenges when it comes to the systematic assessment of the reliability of health information and unreliability has a negative impact. The use of social media requires analytical skills to evaluate various information in smart way. Clear and suitable objectives for social media use are required, and there should be an agency responsible, through program development, for producing knowledge-enhancing content to guide the use of social media towards the most useful content.

Therefore, in this research, the researcher had been studying international papers, and research studies were conducted to analyze, synthesize, and constituents of intelligent digital learning platform to enhance digital health literacy after that design an intelligent digital learning platform to enhance digital health literacy and make out the platform assess the suitability of intelligent digital learning platform to enhance digital health literacy so as to develop in the next step.

\section{Theoretical background}

\subsection{Digital health literacy}

Digital health literacy is a set of skills relating to an individual in terms of accessing, understanding, and using digital health information effectively, to provide a basis for health learning and practices. The promotion of digital health literacy can lead to longterm development, resulting in better health outcomes and chronic disease prevention. [2] It is the necessary skill for guiding and implementing digital health technology and integrating digital technology into modern healthcare methods, known as digital health. [3] This is a complement of digital health literacy in the context of potential technology, to assist in health literacy promotion and building up the computational and literacy skills needed for health literacy promotion, so that healthcare professionals can communicate with technology that allows patients to participate in sharing decisions. The emerging technologies can help them to obtain the data processing of health information and basic health services, which are essential for appropriate health decisions, contributing to ongoing plans while gaining greater privacy. [4] Moreover, they can gain the ability to search, understand, exchange, and evaluate health information from an online environment. Later, they can apply the knowledge gained of medical treatment to treat health problems [5].

In conclusion, digital health literacy is a set of basic skills acquired through effective digital technology to search, understand, and evaluate an individual's health information. This relates to the use of digital health technology from the online environment and to applying the knowledge acquired. 


\subsection{Digital learning platform}

A Digital Learning Platform is the application of technology to learning management to keep up with any changes and encourage people to seek knowledge by themselves, from digital media and social media. In the digital age, people have the ability to create and develop learning innovation to meet the needs of self-learning by the creation of a free social learning platform. The registration of their identity means they can access and make use of platforms such as Website, Fan page, Facebook, Line Open Chat, LINE Official Account, Google Sites, Instagram, Twitter, YouTube, etc. The digital learning platform has become a learning trend in the digital age. Anyone can be both a learner and an educator and generate a large amount of information that can be analyzed and processed into new knowledge and disseminated through social media. It is an architecture that integrates platforms with digital systems. Digital platforms are not limited only to websites or applications. They include all platforms that are the Touchpoints of the target audience. This may include websites, campaign sites, social media, Mobile Sites, Mobile Applications, SMS, E-Mail, Search Sites, GeoSocial Sites, Web TV, Digital Video / Audio and Games. These often have a core infrastructure, such as a CRM (Customer Relationship Management) system to record and analyze user behavior and providing a tailored experience to the target audience. It can analyze learning data to gain insights and quantitative data. The overview platform ranges from a set of hardware to the analytic system. [6] The connections are between computers that transfer data between each other, which can be connected to another computer system called a server, used for control and guidance of choice; for example, the priorities for searching news, news feeds, or diverse opinions on consumer timelines and posting of information that is required to recheck the information. The platform needs to be transparent in the step-by-step instructions or conditions that will allow the robot to do the things assigned [7].

In conclusion, a digital learning platform refers to a technology that has been designed for insight analysis of learning management, as well as the ability to control and assist by having divergent infrastructure systems to meet the needs of self-learning skills through the creation of a learning platform from online social media.

\subsection{Intelligent technology to enhance learning}

The growth of digital information and advances in computing influences have made significant advances in learning for clinical decision support, treatment diagnosis and prognosis. These advances inspire the hope of better medical care and treatment. The smart technologies for supportive learning include:

Decision support system. This is software that helps with decision making. The information of the individual was matched with the clinical database with the computer system. A database and knowledge management can contribute to decision support systems in healthcare processes. The advantages of this system can aid personalized care by filtering individual data and intelligently presenting it at the right time, for example, reminders about essential chronic disease management services, online advice on patient screening, a personal health record system, etc. [8] At present, the knowledge of 
data mining is used in various research areas, including medical research. Data mining is the process of searching for information or knowledge contained in large, complex databases in order to use the knowledge gained in decision-making. Data mining is an evolution of data interpretation from simple data storage into a database [9].

Data classification. The data classification process involves learning and learning classification. Learning information is analyzed by a classification algorithm. The test data has been classified to assess the validity of the classification rules. If the validity is acceptable, the rules can be used with new information. It is easy to analyze closed questions in questionnaires, but not easy for open-ended questions to be adjusted to fit classification rules, because the question is complex and inaccurate due to bias from data analysts. Data mining is the computerized technique for discovering the knowledge base of big data with statistical principles [10].

Security server. Data protection and the security of the database are provided to those involved only in the interests of security. The Microsoft SQL Server database system is a relational database management system type client-server with a real-time output of Visual Basic programming. Data storage is managed with an SQL Server database management program based on the system's stability, reliability, and maximum security, with ISO 27001: 2013 as standard [10].

Artificial intelligence. The designing of healthcare applications by using artificial intelligence could help alleviate the shortage of doctors to meet the needs of an aging population and inequality in access to healthcare in low-resource countries. In the design of artificial intelligence technology, safety is critical for healthcare applications. The complex healthcare systems that the physicians work in need to perform good judgment and ethical responsibility to gain the trust of patients. They tend to believe in doctors treat them with goodwill. Since the patient wants to know why they are followed Artificial Intelligence systems, it can only be said that Artificial Intelligence can give recommendations. [11] The general principles for designing Artificial Intelligence are: 1) combine population data to select demographic groups, which is the first requirement of a studied population and environment, including patient demographics and cohort selection. It is important to know the target population; 2) combine the population data to compare with the population that can be applied with the model. These details can help to estimate the potential prejudices and equality issues. A detailed document of patient characteristics should be prepared. The sensitive variables in the population, such as race and socioeconomic status, should also be documented. Data transparency is essential to foster fairness and equitable models; 3) define details of architecture, models, and model development objectives to compare with designed models. Other important details include modeling. Property Selection Techniques and the management of missing values should be transparent in the proper implementation of the Artificial Intelligence model in healthcare, and 4) transparency in the model evaluation report, model optimization and validation. The evaluation of the model should define a detailed strategy, as well as the guidelines that are used for evaluation. It is necessary to define the options of validation metrics, such as sensitivity, specificity, positive prediction values or the area under the curve, and receiver performance. As a final stage, transparency assessment is essential for healthcare to achieve and maintain the confidence and trust of all stakeholders [12]. 
A database. A database is a data storage space designed for the efficient storage of data. It allows users to manage their data retrieval and gain access to information in a variety of ways and includes performing data maintenance. There are many types of databases, such as relational databases, hierarchical databases, and networked databases. [10] Many websites have fully implemented a database with the computer and many kinds of database software have been invented. Nowadays, computers are used to store data with a general commercial program. Users do not have to do programming; they simply must learn the commands to retrieve data or manage data, such as entering data, data storing, editing, and changing data, etc. The important factor of the database is the acquisition of accurate, complete, and rapid information by operating the information system as a tool to transform the data into the form of information that is ready and available for use. The information must be up to date, with minimal data redundancy and data sharing.

Forecasting. This is the analysis of structural equations to forecast and analyze multi variance problems so that researchers can answer research questions or objectives. They can use statistics effectively and can confirm or draw conclusions from the causal correlation. At present, multivariate analysis techniques are widely used in research. [13] Structural equation modeling analysis has some steps, as follows: 1) analyzing the confirmation elements; 2) testing the correlation between observable variables, and 3) analyzing the structural equation model analysis.

The deep neural structure. The deep neural structure is a branch of Machine Learning. The fundamentals of deep learning are the algorithm that tends to create a model to represent data meaning at a high level. The creating of a data architecture is combined with several substructures that are derived from non-linear transformations. [13] For example, one picture can be represented as a vector of brightness per pixel point. The representation of meaning that makes the learning for performing tasks is easier than ever. Facial recognition or facial expression recognition regarding deep learning is considered a method with a lot of potential for addressing non-instructor learning features or Semi-Supervised Learning.

Cloud storage. Cloud data processing is transforming the current design of enterprise IT infrastructure. It is an IT service solution that can be implemented as needed, with the flexibility to be scaled up and down as needed. [14] Users can save their information in the cloud storage. Data access is completed by connecting to Networks and Client Services. The advantage of cloud storage is that data can be accessed from anywhere. The cloud has four basic features: [15] 1) flexibility: the user can access for service or storage as needed; 2) it can be operated manually and automatically cancel of access; 3) a connection channel between one website and another website, and 4) billing based on the service. The advantages of cloud storage are flexibility, accessibility, and reliability. It provides better protection, reduces the problems caused by hardware failure, has low cost, and is suitable for small to medium-sized applications. It is flexible, scalable, seamless, and efficient, it supports growth, it is cost-effective with pay-as-you-go models, it can support a wide variety of platforms including mobile browsers and more, and this makes applications accessible. There is no need for technical experts to maintain storage and one can allow others to access the data, resulting in a collaborative model [14], [15]. 
Interactive digital tools. These are a combination of a variety of devices, such as head-mounted computers, monitors, body sensors, device-specific interfaces, real-time graphics, and sensory rendering systems in content design, which are presented with computer-generated graphics to attract users. A virtual world is created that changes naturally with head and body movements. The outside world is replaced by the perception of a simulated environment. There are other forms of digital technology, such as interactive digital games, that can be created to manage healthcare education and health-related behavior change. It is a new option for the dissemination of healthcare information. Promotion and other health-related activities may affect attitudes, changing users' behavior in the engagement [16] and affecting users' awareness, motivation, and immersion. Interactive digital tools encourage audiences to view additional exhibits for a longer time [17].

\section{$3 \quad$ Research methodology}

The research method was divided into three phases according to the research objectives as follows:

- Phase 1: Phase 1, an international synthesis of documents and research of an intelligent digital learning platform to enhance digital health literacy. It is the study and research related to characteristics of a person with digital health literacy, Guidelines for developing people to acquire digital health literacy, component of a digital learning platform. Published in the international research base system from 2011-2021, there were a total 27 issues. Which is shown in the essay diagrams in Figures .3-1 The research tool was content analysis form and data analysis by content analysis technique.

- Phase 2: Phase 2 draws on information gained from the synthesis stage to design an intelligent digital learning platform to enhance digital health literacy, comprising a characteristics of a person with digital health literacy, guidelines for developing people to acquire digital health literacy, component of a digital learning platform are presented in an illustration plan and in the essay in Figure 4.

- Phase 3: Phase 3 assesses the suitability of an intelligent digital learning platform to enhance digital health literacy. The evaluation was conducted by 10 experts, who were categorized as 5 computer engineering technology experts and 5 medical technology experts with more than 5years of relating experience. After that improves the intelligent digital learning platform to enhance digital health literacy follow the advice of experts. The research instruments were an intelligent digital learning platform to enhance digital health literacy and suitability assessment form. The statistics used for data analysis were a -5level evaluation scale. The statistics used in the data analysis are arithmetic mean and standard deviation. 


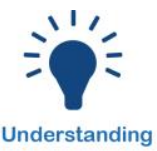

Understanding

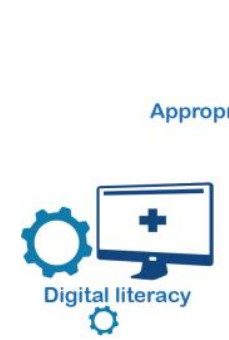

\section{Characteristics of person with} Digital health literacy

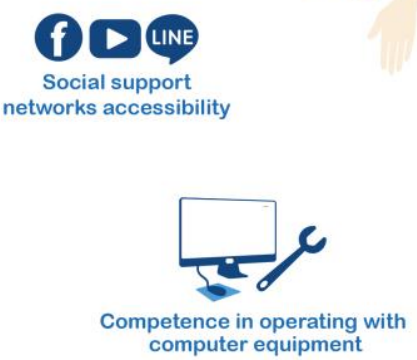

Fig. 1. Characteristics of a person with digital health literacy

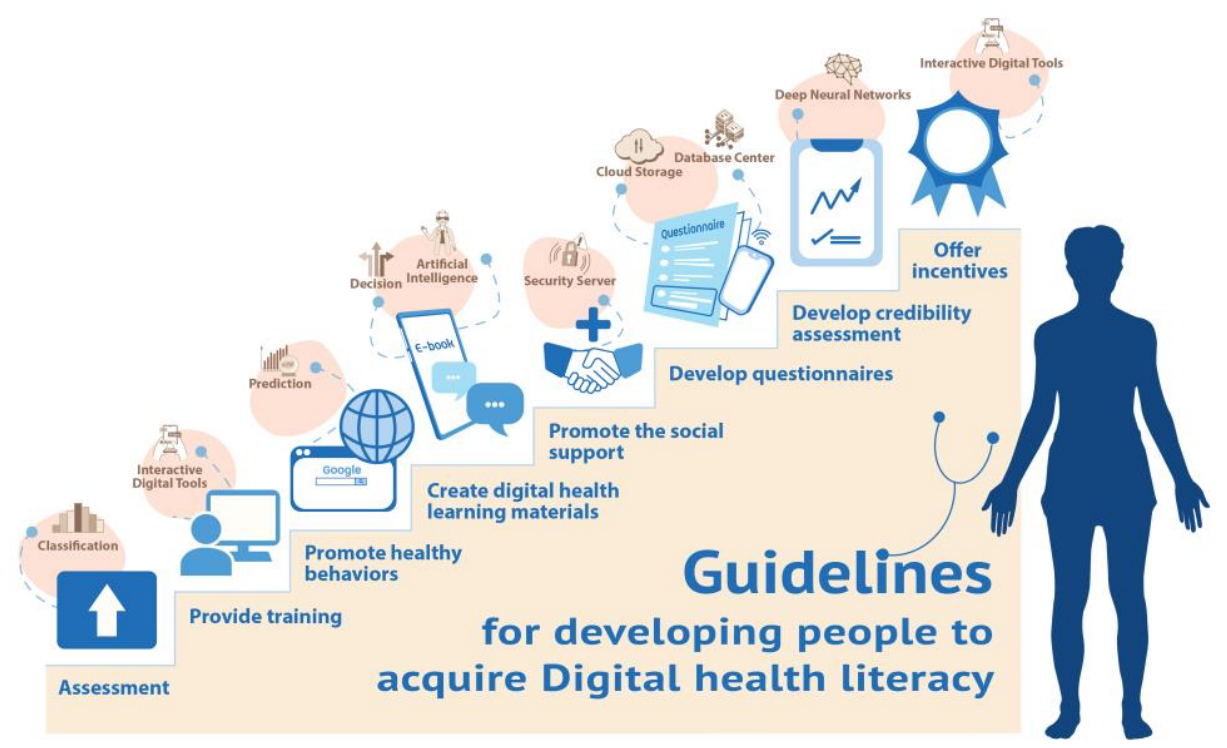

Fig. 2. Guidelines for developing people to acquire digital health literacy 


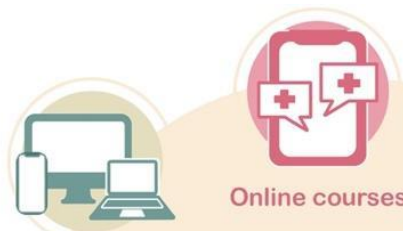

Access devices
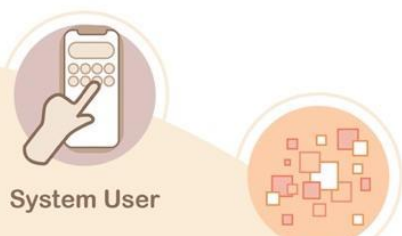

System Technology

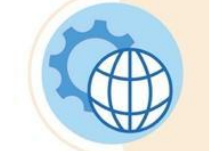

System development standards

Electronic security

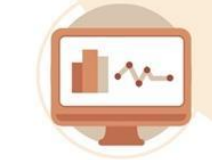

Learning Analytics
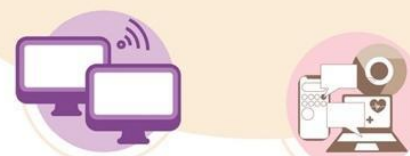

Component of a digital learning platform

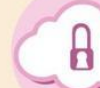

Network System

Usage Type

Fig. 3. Components of a digital learning platform 


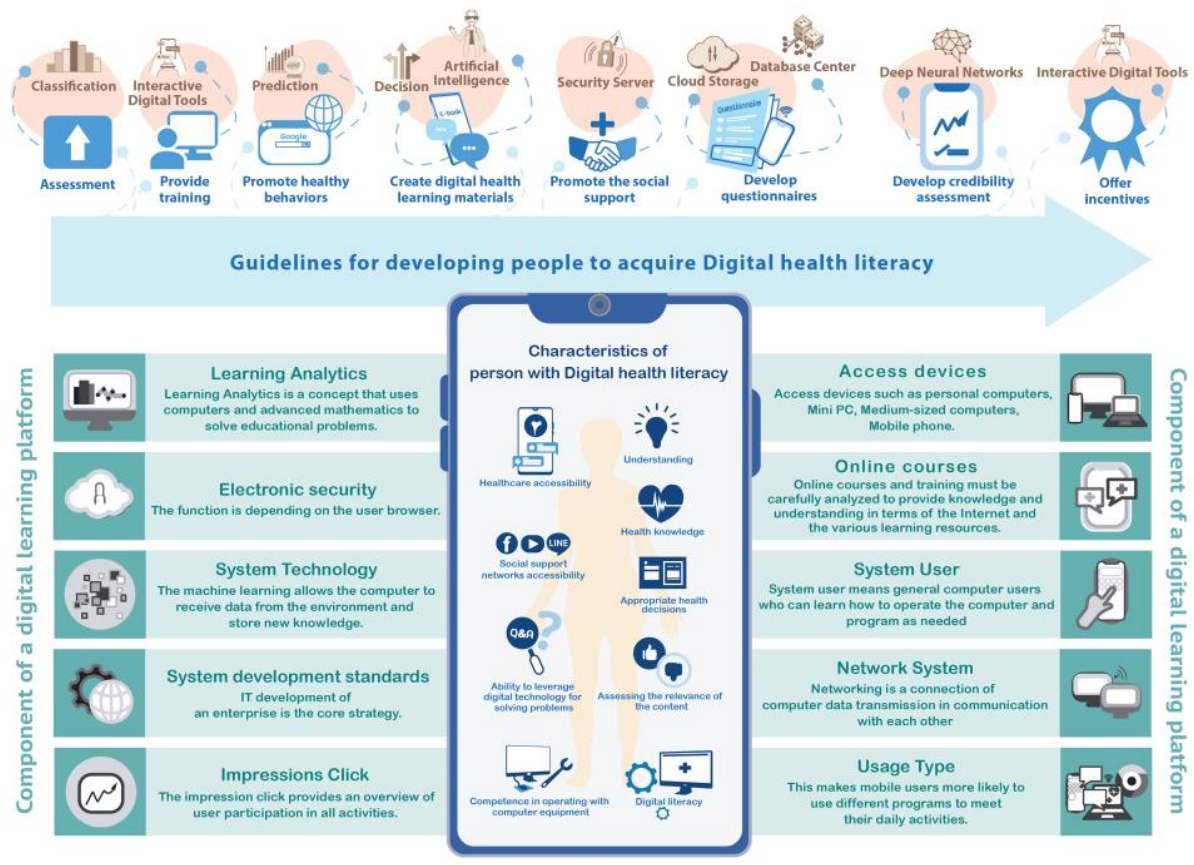

Intelligent Digital Learning Platform to Enhance Digital Health Literacy

Fig. 4. Intelligent digital learning platform to enhance digital health literacy

\section{$4 \quad$ Results}

\subsection{The results of the synthesis documents and research}

Part 1 results of an international synthesis of documents and research related to an intelligent digital learning platform to enhance digital health literacy.

Summary of the characteristics of people with digital health literacy. The nine features of people with digital health literacy [2], [4], [18], [19], [20], [21], [22] are as follows: 1) healthcare accessibility; 2) social support network accessibility; 3) understanding; 4) ability to leverage digital technology to solve problems; 5) Digital literacy; 6) competence in operating computer equipment; 7) health knowledge; 8) assessing the relevance of the content, and 9) making appropriate health decisions, as shown in Figure 1.

Figure 1, shows the characteristics of the person with digital health literacy, including access to comprehensive digital healthcare from beginning to end. The person can access a social support network that connects members, physicians, and healthcare providers together in a sustainable ecosystem. This person has the understanding and ability to leverage digital technology to address local medical care problems wherever they are. They can receive advice with prescription services, while patients can contact their doctors to manage and treat their illness. The person has computer knowledge and the 
ability to manage computer equipment, has health literacy and can use the online healthcare system, which can provide an opinion regarding holistic healthcare. The person can make the assessment of the relevance of content pertaining to chronic or critical illness and make appropriate health decisions.

Guidelines for helping people acquire digital health literacy. These guidelines consist of eight main approaches: [2], [3], [4], [20], [21], [22], [23] 1) assessment of information technology accessibility, such as surveying the use of the Internet and computers, their digital skill levels and the types of devices most frequently used, to reduce health inequality and assess them in terms of digital health literacy. Assessing the community's need for Internet access can be a source of supportive data for tackling health issues; 2) providing training to educate healthcare personnel for collaboration with digital health stakeholders in the research process for developing community representatives. The interaction between healthcare providers through the healthcare system has a social influence, resulting in wider learning, such as of law and policy; 3) promoting healthy behaviors to balance the use of technology, by initiating user participation and co-creation, with the objective of combining community preferences; 4) creating digital health learning materials to educate people in remote communities and develop platform tools that improve health skills, such as website development with comprehensive and up-to-date health content, developing large-scale online open sources for online courses, and disseminating information quickly through social networks, digital health learning materials such as artificial intelligence, smart video, audio applications, online publications, websites, etc. 5) promoting the social support to change digital behavior. The level of commitment of individuals influences their well-being. Optimism is key to fostering resilience for positive changes when faced with chronic disease; 6) developing questionnaires on health knowledge, such as a health impact questionnaire, semistructured interviews, perceived health proficiency assessments, health literacy surveys and consent forms, etc. These are used to assess social data, relevance, age differences, education levels and digital health learning skills; 7) developing credibility assessment criteria, assessing their relevance, and increasing the content of online health information to support self-management and self-management activities, and 8) offering incentives, such as financial compensation for participation, as shown in Figure 2.

The Component of a digital learning platform. The digital learning platform is the learning center for learners and personnel to assess their performance in accordance with the standards set by the organization. The compositions of a digital learning platform [6], [10], [12], [24], [25], [26], [27] (as shown in Figure 3) are:

1. Networking, which is the connection of computer data transmissions in communication with each other. Data transmission patterns within the network are divided into two types: 1) Half Duplex: data can be sent and received but cannot be executed simultaneously. If the sender transmits data, the receiver is unable to send it until the sender stops sending the information. Later, it is the sender's turn; 2) Full Duplex: both sender and received can send and receive information simultaneously. It is the same as talking on the phone. Networking is divided into three types as follows: 1) LAN (Local Area Network) is a network connection in nearby areas, such as in university, among units via Ethernet, Fast Ethernet, FDDI, Token Ring; 2) WAN (Wide 
Area Network) is the connection between Lan networks, in case of long connection distances. The connection can be done by using ATM, DSL, ISDN, but it has a lower connection speed than Lan connection, and 3) MAN (Metropolitan Area Network) is a large area computer network connection, like WAN, but the connection has high speed.

2. Usage Type 1) Web Application Technology is the use of web applications that can be accessed by web browsers through a computer network, such as the Internet or Intranet. The advantage of using web applications is the work will be installed on the server to serve the client and the client does not need to install additional programs. They can use browser-type programs that come bundled with the operating system and can be used immediately, and 2) Mobile Applications: smartphone usage has exponentially increased, especially in the development of mobile applications, which can be categorized as system application development and software applications that respond to the usage of the device. This makes mobile users more likely to use different programs to meet their daily activities.

3. Access devices, such as personal computers, Mini PC, Mobile phones, Mediumsized computers, etc.

4. System technology consists of 1) an interconnection channel from Application Programming Interface (API), which is an intermediary that allows an application to connect with another application or connect to the operating system, allowing data to be transmitted across servers, and 2) a big data management system to search for insights. This enables the analysis of large and very complex data, which is beyond the scope or capabilities of traditional data manipulation. The data can be recorded, managed, and processed in a timely manner. Machine learning allows the computer to receive data from the environment and store new knowledge as a base for the replacement of any knowledge.

5. There are two common types of electronic security. Secure Sockets Layer (SSL) is a standard security protocol for bridging an encrypted link between a web server and a browser in online communications. The other is Transport Layer Security (TLS), which is a protocol developed on the basis of SSL. The difference between TLS and SSL is the encryption process. The encryption of SSL requires individual use of the encrypted and unencrypted data ports. TLS can encrypt from encrypted and nonencrypted data ports. The function is dependent on the user's browser, whether it supports TLS or not. If not, the encryption will be SSL.

6. Learning Analytics (LA) is a concept that uses computers and advanced mathematics to solve educational problems. The key personnel for LA are three in number: 1) An educator with a broad spectrum of development, psychology, medical knowledge, brain functions, social culture, politics, and learning; 2) a data scientist, and 3) a specialist in big database management and algorithms. LA consists of four steps: step 1 is data collection, step 2 is diagnostics, step 3 is forecasting, and step 4 is Learning Design.

7. System development standards: the IT development of an enterprise is the core strategy. An IT system functions based on the knowledge and potential of the software system, server system, computer network system and, most importantly, the backup system. The data center is the heart of an organization's computer and information 
systems. The primary function of the data center is to maintain the stability of the organization's IT system in order to provide uninterrupted service and to improve the existing data centers, so they are more efficient and suitable for implementation. The information system is divided into six types, as follows: 1) the transaction processing system is an information system that stores and processes the routine information of the organization; 2) the management information system, which is a system that brings information from various sources to prepare reports for planning control of the organization's operations; 3) the decision support system. This is an information system that creates decision-making guidelines for users by creating a decision-making scenario for each choice. Therefore, the users can be confident in making the decisions as needed; 4) the expert system. This is an information system that simulates human decisions with expertise in a specific area. The expert system makes decisions for the user by imitating the rational human decisions made from real experience and gathering the knowledge base of experts as information for decision making. Most of them will be used to solve difficult problems that require specialized knowledge; 5) office automation systems. These are the systems which support office activities, facilitate the documentation in the organization to perform functions more efficiently and 6) the information system for senior management. This is a system designed to meet the needs of senior management. The system is user friendly, and it can forecast and analyze trends related to policy controls and the strategic planning of senior management by working with a decision support system.

8. The term 'system user' means general computer users who can learn how to operate the computer and program as needed. The users can be divided into 1) computer care and maintenance staff who have always checked the availability of the computer at all times; 2) the computer programmer, who analyzes computer systems in order to obtain programs that meet the objectives of use in the organization; 3 ) the system designer and analyzer, who is the person responsible for determining what kinds of computer the organization should use for maximum benefit and good quality, and 4) the computer system administrators, who are responsible for managing all kinds of computer-related resources for maximum benefit to the organization.

9. The impressions click is the click-through rate, which is displayed as a percentage to indicate how many percent the user clicked on the results page. The impression click provides an overview of user participation in all activities on the digital learning platform. It reflects the interest of the system users.

10. Online courses, online learning management and training must be carefully analyzed to provide knowledge and understanding in terms of the potential of the Internet and the various learning resources on the Internet. Generally, the design is based on the ADDIE Model Principle, which is a teaching style development process commonly used by instructional designers and training developers. There is a 5-stage development sequence: 1) the analytical stage, which is the understanding of teaching and learning problems, the objectives of the teaching style and the objectives that will be created, as well as the learning environment, basic knowledge and skills required by the learner; 2) the design phase, which consists of creating learning objectives, defining the assessment tools, exercises, content and teaching plan, and selecting the teaching materials; 3 ) the development phase, which is the design of each part of the 
assessment tools, creating exercises, creating content and program development for teaching materials. Later, the results are tested to find errors and make improvements; 4) implementation: the objective of this step is for effective and efficient teaching, to promote the learner's understanding and support the learner with the required learning objectives, and 5) the assessment, which consists of two parts: model assessment and overview result assessment.

\subsection{Platform design}

In this step, it is the design of an intelligent digital learning platform to enhance digital health literacy, comprising a characteristics of a person with digital health literacy, guidelines for developing people to acquire digital health literacy, component of a digital learning platform are presented in an illustration plan and in the essay in Figure 4.

\subsection{The evaluation results of an intelligent digital learning platform}

Part 3 Assessment results of an intelligent digital learning platform to enhance digital health literacy.

The results of the assessment of the suitability of an intelligent digital learning platform to enhance digital health literacy. The results of the evaluations of each component can be summarized as follows: 1) Characteristics of people with digital health literacy this was found to be the most suitable (Mean $=4.68$, S.D. $=0.21$ ); 2 ) Guidelines for helping people acquire digital health literacy this was found to be the most suitable (Mean $=4.76$, S.D. $=0.26) ; 3$ ) The Component of a digital learning platform this was found to be the most suitable (Mean $=4.84$, S.D. $=0.21$ ); 4) Overall design suitability of intelligent digital learning platform to enhance digital health literacy this was found to be the most suitable (Mean $=4.95$, S.D. $=0.22$ ), and when applying the results of the evaluation of various components to find the combined mean of all the evaluations of an intelligent digital learning platform, this was found to be the most suitable (Mean = 4.78, S.D. $=0.23$.

\section{Conclusion and discussion}

Digital health literacy is a powerful set of basic and effective skills to explore, understand and assess individual health information in digital health from an online environment, in order to apply the acquired knowledge to improve health, which leads to ongoing long-term development and chronic disease prevention. The skills development approach to enhance digital health literacy should be able to generate knowledge through diverse digital media. Digital learning is useful for people in the digital age who can be both learner and educator, disseminating knowledge via social media. In the management of smart digital learning to enhance digital health knowledge, there are important elements that need to be prepared for: 1 ) survey needs; 2) preparing health 
literacy information; 3) access channels; 4) data storage; 5) data usage; 6) Data Analysis; 7) data management, and 8) networking. When integrating different systems to disseminate information to different parts, the responsible department should recognize the importance of developing digital skills to the personnel using information technology in their work as a useful tool.

Intelligent Digital Learning Platform to Enhance Digital Health Literacy from the integration of information and communication technology to promote learning with interactive teaching and learning. The learner can control the learning and can create a knowledge base by themselves. Today, different platforms have been created, and there are different approaches to access content. The learning process is constantly always changing on every level. The trend of the learning process is the result of living with smart machines. Therefore, building a smart digital learning platform to enhance digital health literacy should be concerned with the content, which will be in line with the new knowledge base. Because education is controlled by programming, educational personnel must have sufficient knowledge to keep up with technology and always develop their own potential.

An Intelligent Digital Learning Platform to Enhance Digital Health Literacy involves the transformation of education from the traditional system into a new and modern process. The benefits that the organization will receive are divided into three areas: 1) Education. The management of online teaching and learning for the education industry has gradually adjusted. But with the Coronavirus 2019 pandemic, the use of technology in the management of online teaching has accelerated. It is important that "communication systems" have a corresponding understanding between "administrators and teachers", "teachers" and "teachers and students". It can raise the quality of modern education to improve standards and meet the needs of the target group; 2) Health rehabilitation. Thailand has witnessed a noticeable increase in the elderly population. The future aging population will be different from the past. In the future, old people will have a better education. They will have an active lifestyle and will be looking for digital information on health. A smart digital learning platform for healthcare will help them to monitor healthcare services by themselves. A virtual assistant is available for consultation; 3) Training. Students can learn from anywhere at a convenient time. Training arrangements should include a variety of activities to understand and overcome the challenges of each learner's situation. The lecture should focus on the content that is close to learners. In this way, it will be easy to learn. In organizing activities, learners should be encouraged to use what was taught when exchanging their learning outcomes. They can practice expression and work together as a team. The platform acts as a learning facilitator, driving development by the learner's characteristics. Teachers and learners can engage, communicate, interact, and share media as if they were in a real classroom. Once the learners have passed the required criteria, they can request a certificate through the training system. To advance in the field of work, they can develop existing knowledge and learn new things for the purpose of both organizational and self-development. 


\section{Acknowledgment}

Thank you Mahidol University, King Mongkut's University of Technology North Bangkok and Rajamangala University of Technology Rattanakosin (Wang Klai Kangwon Campus) for supporting this study and thank you to all the experts for evaluating the platform in this research.

\section{$7 \quad$ References}

[1] S. Kaeophanuek, J. Na-Songkhla, and P. Nilsook, "A learning process model to enhance digital literacy using critical inquiry through digital storytelling (CIDST)," Int. J. Emerg. Technol. Learn., vol. 14, no. 3, pp. 22-37, 2019. https://doi.org/10.3991/ijet.v14i03.8326

[2] A. Hyman et al., "Testing a school-based program to promote digital health literacy and healthy lifestyle behaviours in intermediate elementary students: The Learning for Life program," Prev. Med. Reports, vol. 19, no. June, 2020. https://doi.org/10.1016/j.pmedr .2020 .101149

[3] A. Shi, M. Rajpal, and P. Kostoff, "Validation and psychometrics for the Health Skills Profile," Heliyon, vol. 5, no. 10, pp. 4-8, 2019. https://doi.org/10.1016/j.heliyon. 2019.e02558

[4] P. Dunn and S. Conard, "Improving health literacy in patients with chronic conditions: A call to action,” Int. J. Cardiol., vol. 273, no. August, pp. 249-251, 2018. https://doi.org/ $\underline{10.1016 / j . i j c a r d .2018 .08 .090}$

[5] U. H. Bittlingmayer, K. Dadaczynski, D. Sahrai, S. van den Broucke, and O. Okan, "Digitale Gesundheitskompetenz - Konzeptionelle Verortung, Erfassung und Förderung mit Fokus auf Kinder und Jugendliche," Bundesgesundheitsblatt - Gesundheitsforsch. - Gesundhe itsschutz, vol. 63, no. 2, pp. 176-184, 2020. https://doi.org/10.1007/s00103-019-03087-6

[6] R. McConville et al., "Vesta: A digital health analytics platform for a smart home in a box," Futur. Gener. Comput. Syst., vol. 114, pp. 106-119, 2021. https://doi.org/10.1016/j.fu$\underline{\text { ture.2020.07.046 }}$

[7] C. Marsden, T. Meyer, and I. Brown, "Platform values and democratic elections: How can the law regulate digital disinformation?," Comput. Law Secur. Rev., vol. 36, 2020. https:// doi.org/10.1016/j.clsr.2019.105373

[8] M. M. Paulsen, C. Varsi, and L. F. Andersen, "Process evaluation of the implementation of a decision support system to prevent and treat disease-related malnutrition in a hospital setting,” BMC Health Serv. Res., vol. 21, no. 1, pp. 1-14, 2021. https://doi.org/10.1186 /s12913-021-06236-3

[9] M. C. Massi, F. Ieva, and E. Lettieri, "Data mining application to healthcare fraud detection: A two-step unsupervised clustering method for outlier detection with administrative databases," BMC Med. Inform. Decis. Mak., vol. 20, no. 1, pp. 1-11, 2020. https://doi.org/ 10.1186/s12911-020-01143-9

[10] P. Pornpongtechavanich and P. Wannapiroon, "Intelligent Interactive Learning Platform for Seamless Learning Ecosystem to Enhance Digital Citizenship's Lifelong Learning,” Int. J. Emerg. Technol. Learn., vol. 16, no. 4, pp. 232-248, 2021. https://doi.org/10.3991/ijet. v16i14.22675

[11] I. Habli, T. Lawton, and Z. Porter, "Artificial intelligence in health care: Accountability and safety," Bull. World Health Organ., vol. 98, no. 4, pp. 251-256, 2020. https://doi. org/10.2471/BLT.19.237487 
[12] T. Hernandez-Boussard, S. Bozkurt, J. P. A. Ioannidis, and N. H. Shah, "MINIMAR (MINimum information for medical AI reporting): Developing reporting standards for artificial intelligence in health care," J. Am. Med. Informatics Assoc., vol. 27, no. 12, pp. 2011-2015, 2020. https://doi.org/10.1093/jamia/ocaa088

[13] W. Ding, J. Nayak, H. Swapnarekha, A. Abraham, B. Naik, and D. Pelusi, "Fusion of intelligent learning for COVID-19: A state-of-the-art review and analysis on real medical data," Neurocomputing, vol. 457, pp. 40-66, 2021. https://doi.org/10.1016/j.neucom.2021.06.024

[14] R. A. P. Rajan, "Evolution of Cloud Storage as Cloud Computing Infrastructure Service," IOSR J. Comput. Eng., vol. 1, no. 1, pp. 38-45, 2012.

[15] S. Obrutsky, "Cloud Storage: Advantages, Disadvantages and Enterprise Solutions for Business," EIT New Zeal., no. July, 2016, [Online]. Available: https://www.researchgate.net/publication/305508410_Cloud_Storage_Advantages_Disadvantages_and Enterprise_Solutions_for_Business.

[16] A. Rizzo, B. Lange, E. A. Suma, and M. Bolas, "Virtual reality and interactive digital game technology: New tools to address obesity and diabetes," J. Diabetes Sci. Technol., vol. 5, no. 2, pp. 256-264, 2011. https://doi.org/10.1177/193229681100500209

[17] S. Rizvic, A. Sadzak, V. Hulusic, and A. Karahasanovic, "Interactive digital storytelling in the sarajevo survival tools virtual environment," Proc. - SCCG 2012 28th Spring Conf. Comput. Graph., no. November 2016, pp. 109-116, 2012. https://doi.org/10.1145/2448531 $\underline{.2448545}$

[18] M. J. Haenssgen, N. Charoenboon, and G. Zanello, "You've got a friend in me: How social networks and mobile phones facilitate healthcare access among marginalised groups in rural Thailand and Lao PDR,” World Dev., vol. 137, 2021. https://doi.org/10.1016/j.worlddev .2020 .105156

[19] K. Harris, G. Jacobs, and J. Reeder, "Health Systems and Adult Basic Education: A Critical Partnership in Supporting Digital Health Literacy," Heal. Lit. Res. Pract., vol. 3, no. 3, pp. S33-S36, 2019. https://doi.org/10.3928/24748307-20190325-02

[20] L. Perestelo-Perez et al., "IC-health project: Development of MOOCs to promote digital health literacy: First results and future challenges," Sustain., vol. 12, no. 16, 2020. https://doi.org/10.3390/su12166642

[21] W. Sui and D. Facca, "Digital health in a broadband land," Heal. Sci. Inq., vol. 11, no. 1, pp. 140-143, 2020. https://doi.org/10.29173/hsi294

[22] R. van der Vaart et al., "The role of age, education, and digital health literacy in the usability of internet-based cognitive behavioral therapy for chronic pain: Mixed methods study," JMIR Form. Res., vol. 3, no. 4, pp. 1-11, 2019. https://doi.org/10.2196/12883

[23] A. Banbury et al., "Adding value to remote monitoring: Co-design of a health literacy intervention for older people with chronic disease delivered by telehealth - The telehealth literacy project," Patient Educ. Couns., vol. 103, no. 3, pp. 597-606, 2020. https://doi.org/10. 1016/j.pec.2019.10.005

[24] P. Palee, P. Wannapiroon, and P. Nilsook, "The Architecture of Intelligent Career Prediction System based on the Cognitive Technology for Producing Graduates to the Digital Manpower," Int. J. Adv. Comput. Sci. Appl., vol. 11, no. 12, pp. 115-121, 2020. https://doi.org/10.14569/IJACSA.2020.0111214

[25] M. J. Sousa and Á. Rocha, "Digital Learning in an Open Education Platform for Higher Education Students,” EDULEARN18 Proc., vol. 1, no. November, pp. 11194-11198, 2018. https://doi.org/10.21125/edulearn.2018.2770

[26] A. Mujica-luna, E. Villanueba, and M. L. Lodeiros-zubiria, "Micro-learning Platforms Brand Awareness Using Social- media Marketing and Customer Brand Engagement," vol. 16, no. 17, pp. 19-41, 2021. https://doi.org/10.3991/ijet.v16i17.23339 
Paper-An Intelligent Digital Learning Platform to Enhance Digital Health Literacy

[27] T. Phuthong, "Antecedents Influencing the Adoption of Collaborative Learning Social-Media Platforms Among Thai University Students During the Covid-19 'New Normal' Era," Int. J. Emerg. Technol. Learn., vol. 16, no. 13, pp. 108-127, 2021. https://doi.org/10.3991 lijet.v16i13.18083

\section{Authors}

Kawitsara Eumbunnapong is currently an academic in the production of medical media at Faculty of Medicine Siriraj Hospital Mahidol University. Her research interests include production of medical instructional materials, medical drawing, patient imaging and technology for education (email: kawitsara.kra@ mahidol.edu).

Panita Wannapiroon is an associate professor in the Division of Information and Communication Technology for Education (DICT), Faculty of Technical Education, and Director of Innovation and Technology Management Research Center (ITMRC), Science and Technology Research Institute (STRI), King Mongkut's University of Technology North Bangkok, Bangkok, Thailand (email: panita.w@fte.kmutnb.ac.th).

Phisit Pornpongtechavanich is currently a lecturer at the Department of Information Technology, Faculty of Industry and Technology, Rajamangala University of Technology Rattanakosin Wang Klai Kangwon Campus (RMUTR_KKW), Thailand. His research interests include Security, Deep Learning, Artificial Neural Networks, Deep Neural Network, VoIP quality measurement, QoE/QoS, 3G/4G/5G, Mobile Networks and Multimedia Communication.

Article submitted 2021-10-28. Resubmitted 2021-12-21. Final acceptance 2021-12-23. Final version published as submitted by the authors. 